\title{
ACIDENTES OFÍDICOS NO MUNICÍPIO DE VALENÇA, RIO DE JANEIRO: POSSÍVEL EMERGÊNCIA DE ENVENENAMENTOS POR CASCAVEL
}

\author{
Luiz Henrique Conde Sangenis ${ }^{122}$, Luciano da Silva Lima², Gilberto Ribeiro Leite2, \\ Vanessa Silva Calheiros ${ }^{2}$ Natália dos Santos Rocha² e Marco Antonio Prates \\ Nielebock ${ }^{3}$
}

\section{RESUMO}

O estado do Rio de Janeiro notifica cerca de 500 casos de acidentes por ofídios peçonhentos ao ano. O acidente relacionado com cascavel representa menos de 3\% do total. O município de Valença, situado na região sul do estado, apresentou um aumento do número de notificações de acidentes por serpentes do gênero Crotalus nos últimos anos. Os dados foram coletados do livro de registro da emergência e dos prontuários médicos dos pacientes atendidos no Hospital da Faculdade de Medicina de Valença no período de janeiro de 2003 a julho de 2010. Neste período ocorreram 97 acidentes por ofídios peçonhentos, 49 por Bothrops (50,5\%) e 48 por Crotalus (49,5\%). Após 2005 , houve aumento dos acidentes por cascavel, chegando a 70\% em 2009. Quanto à origem, $81 \%$ ocorreram em área rural. Predominaram os pacientes do sexo masculino $(72 \%)$ e na faixa etária de 15 a 44 anos. Os acidentes por Bothrops, em sua maioria, foram classificados como leves ou moderados e pelo gênero Crotalus, como moderados ou graves. O número de internações foi maior nos casos de acidentes por Bothrops. Embora não tenhamos registrado nenhum óbito, há o risco de aumento de casos fatais, pois o acidente por cascavel apresenta maior letalidade. Dados recentes demonstram a ocorrência de acidentes por Crotalus durissus em outros municípios do sul do estado, o que nos faz concluir que esta serpente continua a se difundir e serve de alerta à vigilância epidemiológica estadual e dos municípios afetados.

DESCRITORES: Acidentes ofídicos; .

1 Instituto de Pesquisa Clínica Evandro Chagas, Fundação Oswaldo Cruz, Rio de Janeiro, Brasil.

2 Hospital Escola Luiz Gioseffi Jannuzzi, Faculdade de Medicina de Valença, Rio de Janeiro - Brasil.

3 Universidade Estácio de Sá, Rio de Janeiro, Brasil.

Endereço para correspondência: Luiz Henrique Conde Sangenis. Instituto de Pesquisa Clínica Evandro Chagas, Fundação Oswaldo Cruz. Avenida Brasil, 4365, Manguinhos, Rio de Janeiro, RJ, Brasil, CEP: 21040-360. E-mail: lhcsangenis@gmail.com

Recebido para publicação em: 6/3/2012. Revisto em: 4/12/2012. Aceito em: 5/2/2013. 


\section{ABSTRACT}

Snake bites in the Municipality of Valença, Rio de Janeiro, Brazil: possible emergence of envenoming by rattlesnake

The state of Rio de Janeiro recorded around 500 notified cases of bites by venomous snakes each year. Accidents by rattlesnake are rare, representing less than $3 \%$ of the total. The municipality of Valença, located in the southern of Rio de Janeiro State, showed an increase in the number of accidents by rattlesnake in recent years. Data presented here were collected from the registry of emergency and medical records of patients treated at the Hospital of the Medical School of Valença in the period from January 2003 to July 2010. In this period there were 97 accidents with venomous snakes, by Bothrops 49 (50.5\%) and by Crotalus 48 (49.5\%). After 2005, there was an increase in accidents by rattlesnake reaching $70 \%$ in 2009 . As to origin, $81 \%$ occurred in rural areas. There was a predominance of males aged 15 to 44 years old. Most accidents by Bothrops were classified as mild or moderate and by the genus Crotalus as moderate or severe. The number of hospitalizations was higher in the genus Bothrops. Although we have not recorded any deaths, there is a risk of increased fatalities since accidents for rattlesnake have a higher mortality. Recent data demonstrate the occurrence of accidents with Crotalus durissus in other municipalities in Southern state, which makes us conclude that this snake continues to spread, serving as a warning and a need for epidemiological surveillance of the state and affected municipalities.

KEY WORDS: Snakebites; rattlesnake; epidemiology.

\section{INTRODUÇÃO}

As serpentes do gênero Crotalus (cascavel) caracterizam-se por habitarem regiões áridas, semiáridas e campos abertos de Cerrado da América do Sul $(1,2)$. No Brasil, o acidente por cascavel não chega a $10 \%$ do total de acidentes por serpentes peçonhentas, embora seja o segundo em importância e o que apresenta a maior letalidade $(5,8,13)$.

O estado do Rio de Janeiro está inserido dentro do bioma Mata Atlântica, com um clima úmido e áreas de floresta, características que não são propícias à ocorrência deste gênero de serpente $(1,2,7,13)$, entretanto diversas regiões do estado tiveram sua paisagem alterada em razão do desmatamento.

O município de Valença, situado no vale do rio Paraíba do Sul, faz divisa a noroeste com o estado de Minas Gerais do qual é separado pelo rio Preto. A maior parte de suas florestas foi desmatada para a produção de café no século XIX, tornando-se, posteriormente, em área de pastagens $(1,16)$. Grandes enchentes que ocorreram entre os anos de 1950 e 1967, na região do rio Preto, podem ter contribuído para a dispersão de cascavéis em áreas desmatadas de Valença e de municípios fronteiriços entre Minas Gerais e Rio de Janeiro (1).

O estado do Rio de Janeiro, de 2003 a 2010, notificou cerca de 500 casos de acidentes relacionados com serpentes peçonhentas ao ano, sendo a grande maioria pelo gênero Bothrops (jararaca) com uma média de $96 \%$ do total de notificações. $\mathrm{O}$ acidente por cascavel é raro e representa menos de 3\% dos acidentes 
(6). Observamos que a partir de 2005 houve um aumento significativo na frequência de notificações de acidentes por cascavel no município de Valença. Tal característica é bastante singular, pois difere de todos os outros municípios do estado onde o acidente por jararaca é predominante $(4,6)$.

Este estudo teve como objetivo descrever o perfil epidemiológico dos acidentes por ofídios peçonhentos no município de Valença no período de 2003 a 2010.

\section{MATERIAL E MÉTODOS}

Os dados foram coletados dos livros de registro de atendimento do setor de emergência e de prontuários médicos dos pacientes atendidos no Hospital Escola Luiz Gioseffi Jannuzzi da Faculdade de Medicina de Valença, referência no atendimento de acidentes por animais peçonhentos no município. Tais atendimentos, realizados no período de janeiro de 2003 a julho de 2010, tiveram como diagnóstico acidente ofídico. As seguintes variáveis foram estudadas: idade, sexo, procedência, data do acidente, gênero da serpente, gravidade, terapêutica empregada e desfecho do caso. Foi considerado internação um período de permanência no hospital superior a 24 horas e observação um período inferior a 24 horas. Embora tenha ocorrido recente modificação na nomenclatura de algumas espécies de serpentes peçonhentas no Brasil, utilizamos a denominação Bothrops, ainda adotada pelo Sistema de Informação de Agravos de Notificação (SINAN) do Ministério da Saúde, para nomear os acidentes com jararacas $(2,6,15)$. Foi utilizado o sistema Epi info para tabulação e análise estatística dos dados.

\section{RESULTADOS}

De 2003 a 2010, ocorreram 97 acidentes por ofídios peçonhentos em Valença, 49 por Bothrops (50,5\%) e 48 por Crotalus (49,5\%); entretanto, apesar de grande variação, nota-se um aumento do número de atendimentos a acidentes por cascavel a partir de 2005 (Tabela 1). Quanto à origem, 81\% dos casos ocorreram em área rural, com predomínio de indivíduos do sexo masculino (72\%). A maior parte dos registros ocorreu nos meses mais quentes, entre outubro e abril (Figura 1). A faixa etária predominante foi a dos 15 aos 44 anos (Figura 2). Os acidentes pelo gênero Bothrops, em sua maioria, foram classificados como leves ou moderados e pelo Crotalus, como moderados ou graves, entretanto o número de internações foi maior no caso dos acidentes por Bothrops (Figuras 3 e 4). Não foram registrados acidentes por serpentes dos gêneros Micrurus e Lachesis e nenhum óbito foi observado na casuística estudada. 
Tabela 1. Número de acidentes por ofídios peçonhentos ocorridos em Valença-RJ, no período de 2003 a 2010, distribuídos por gênero de serpente ( $\mathrm{n}=97)$

\begin{tabular}{|c|c|c|c|c|c|}
\hline \multirow{2}{*}{ Ano } & \multicolumn{4}{|c|}{ Gênero de serpente } & \multirow{2}{*}{ Total } \\
\hline & Bothrops & $\%$ & Crotalus & $\%$ & \\
\hline 2003 & 10 & 90,9 & 1 & 9,1 & 11 \\
\hline 2004 & 6 & 85,7 & 1 & 14,3 & 7 \\
\hline 2005 & 8 & 53,3 & 7 & 46,7 & 15 \\
\hline 2006 & 7 & 38,8 & 11 & 61,2 & 18 \\
\hline 2007 & 7 & 41,1 & 10 & 58,9 & 17 \\
\hline 2008 & 5 & 35,7 & 9 & 64,3 & 14 \\
\hline 2009 & 3 & 30,0 & 7 & 70,0 & 10 \\
\hline $2010^{*}$ & 3 & 60,0 & 2 & 40,0 & 5 \\
\hline Total & 49 & 50,5 & 48 & 49,5 & 97 \\
\hline
\end{tabular}

*dados até julho

\section{Bothrops}

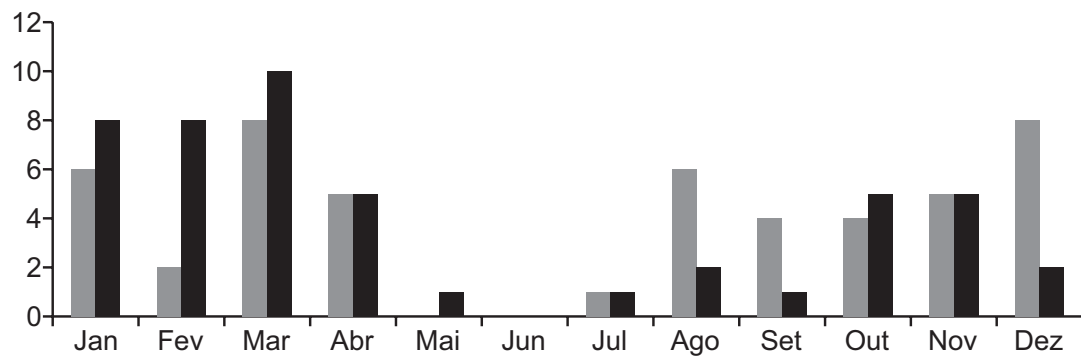

Figura 1. Sazonalidade dos acidentes ofídicos, no período de 2003 a 2010, por gênero de serpente em Valença-RJ ( $\mathrm{n}=97$ ).

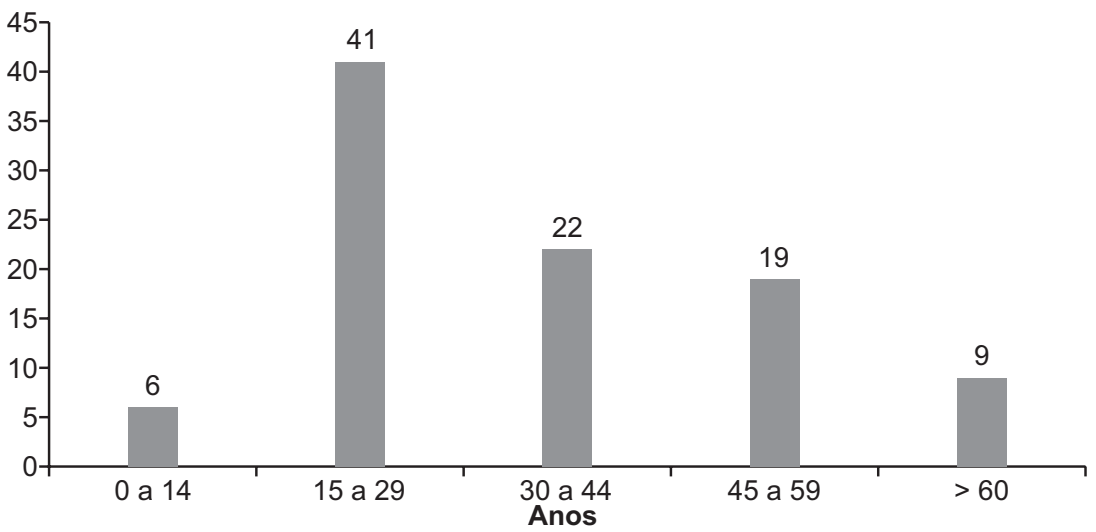

Figura 2. Distribuição dos acidentes ofídicos de acordo com a idade dos pacientes acidentados, no período de 2003 a 2010, em Valença-RJ (n=97). 


\begin{tabular}{|l|l|}
\hline leve & moderado grave \\
\hline
\end{tabular}

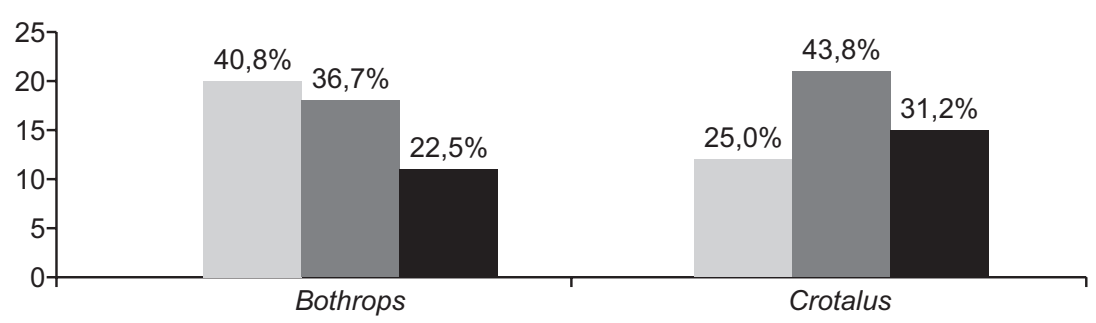

Figura 3. Classificação dos acidentes ofídicos pela gravidade de acordo com o gênero de serpente e suas respectivas frequências, no período de 2003 a 2010, em Valença-RJ ( $n=97)$.

\begin{tabular}{|l|l|}
\hline internação & observação \\
\hline
\end{tabular}

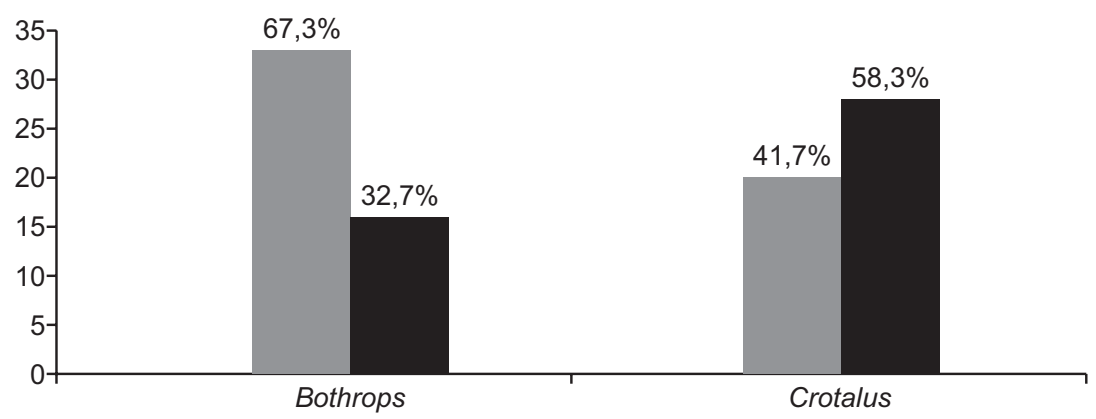

Figura 4. Procedimento adotado nos pacientes vítimas de acidentes ofídicos de acordo com o gênero de serpente, no período de 2003 a 2010, em Valença-RJ ( $\mathrm{n}=97)$.

\section{DISCUSSÃO E CONCLUSÕES}

O que mais motivou a realização deste trabalho foi o fato de o município de Valença apresentar uma ocorrência de acidentes ofídicos diferente dos outros municípios do estado do Rio de Janeiro. Existem registros de acidentes por cascavel em Valença e em outros municípios fluminenses fronteiriços ao estado de Minas Gerais há pelo menos 20 anos (4). Entretanto, o número de acidentes em Valença sempre chamou a atenção por sua maior frequência $(4,6)$. Estes números aumentaram na última década e atualmente os acidentes por cascavel passaram a ser mais notificados do que aqueles por jararaca.

Apenas um trabalho analisou a presença de cascavéis em municípios fluminenses com base em relatos da presença da serpente, capturas, informações 
não oficiais de acidentes e utilizações de soro anticrotálico pelos hospitais. Esse estudo associou a presença de cascavéis aos municípios que fazem fronteira com o estado de Minas Gerais e são banhados pelo rio Preto como Resende, Valença e Rio das Flores (1). Além disso, o desmatamento contribuiu para modificar a paisagem e o clima criando corredores de Cerrado em áreas de Mata Atlântica, o que facilitaria a dispersão e a adaptação da cascavel no estado do Rio de Janeiro (1). É importante notar que estudo epidemiológico realizado anteriormente na região sul do estado de Minas Gerais, área fronteiriça ao sul fluminense, mostrou maior incidência de acidentes por Crotalus do que por Bothrops (8). Dados recentes do SINAN também demonstram uma predominância de acidentes por cascavel no município de Santa Rita da Jacutinga, na Zona da Mata Mineira, que faz divisa com o município de Valença (6). Por outro lado, nota-se que o aumento dos acidentes por cascavel em Valença foi acompanhado de uma redução dos acidentes por jararaca. Talvez o recente aumento do desmatamento no município com redução das áreas de floresta, o crescimento das áreas de pastagem e das zonas urbanas estejam influindo diretamente na epidemiologia desses acidentes $(16,17)$. Outro fator que possivelmente esteja contribuindo para essa redução é a possibilidade de as cascavéis estarem ganhando das jararacas na disputa pelo mesmo nicho biológico (1).

Quanto à sazonalidade dos acidentes, à faixa etária mais acometida, ao predomínio de indivíduos do sexo masculino e à procedência rural, os resultados foram semelhantes aos encontrados em outros estudos epidemiológicos de acidentes ofídicos $(3,5,8,9,10,11,12,14)$. Embora seja mais grave o envenenamento por cascavel, o número de internações foi maior nos casos de acidente por jararaca, provavelmente por ser também maior o número de complicações no local da picada, como abscessos e necrose tecidual $(5,12,14)$. Um fator que poderia explicar a maior gravidade dos acidentes por cascavel é o frequente envolvimento de serpentes adultas neste tipo de acidente (9). Apesar de não termos registrado nenhum óbito em nossa casuística, há o risco de aumento de casos fatais, visto que o acidente por cascavel apresenta maior letalidade por causa da insuficiência renal $(5,13)$.

Analisando dados recentes do SINAN, constata-se que a cascavel continua a se expandir para outros municípios fluminenses que não são banhados pelo rio Preto e nem fazem divisa com o estado de Minas Gerais (6). Acredita-se que o rio Paraíba do Sul e a serra do Mar poderiam servir de barreiras naturais para a expansão da cascavel no estado do Rio de Janeiro. Contudo, Vassouras, Areal e Petrópolis notificaram recentemente acidentes relacionados com esta serpente, $\mathrm{o}$ que talvez contradiga esta hipótese $(1,6)$. Novos estudos devem ser desenvolvidos para confirmar os dados do presente estudo e suas constatações servem de alerta à vigilância epidemiológica do estado do Rio de Janeiro no sentido de adotar medidas de controle e de informação aos serviços de saúde dos municípios afetados.

\section{AGRADECIMENTOS}

Ao Centro de Ensino Superior de Valença (CESVA) e à Faculdade de Medicina de Valença que apoiaram a realização deste estudo. 


\section{REFERÊNCIAS}

1. Bastos EGM, Araújo AFB, Silva HR. Records of the rattlesnakes Crotalus durissus terrificus (Laurenti) (Serpentes, Viperidae) in the state of Rio de Janeiro, Brazil: a possible case of invasion facilitated by deforestation. Rev Bras Zool 22: 812-815, 2005.

2. Bernarde PS. Mudanças na classificação de serpentes peçonhentas brasileiras e suas implicações na literatura médica. Gaz Méd Bahia 81: 55-63, 2011.

3. Bochner R, Struchiner CJ. Epidemiologia dos acidentes ofídicos nos últimos 100 anos no Brasil: uma revisão. Cad Saúde Pública 19: 7-16, 2003.

4. Bochner R, Struchiner CJ. Aspectos ambientais e sócio-econômicos relacionados à incidência de acidentes ofídicos no Estado do Rio de Janeiro de 1990 a 1996: uma análise exploratória. Cad Saúde Pública 20: 976-985, 2004.

5. Brasil. Ministério da Saúde. Fundação Nacional de Saúde. Manual de diagnóstico e tratamento de acidentes por animais peçonhentos $2^{a}$ ed. Brasília: Fundação Nacional de Saúde, 2001.

6. Brasil. Ministério da Saúde. Secretaria de Vigilância em Saúde. Sistema de Informação de Agravos de Notificação (SINAN). Tabulação de dados. Acidentes por Animais Peçonhentos. Disponível em http://dtr2004.saude.gov.br/sinanweb/. Acessado de outubro de 2010 a janeiro de 2012.

7. Brasil. Ministério do Planejamento, Orçamento e Gestão, Instituto Brasileiro de Geografia e Estatística (IBGE). Mapa de Biomas e de Vegetação. Disponível em http://www.ibge.gov.br/home/ presidencia/noticias/21052004biomashtml.shtm. Acessado em novembro de 2011.

8. Franco LR, Rocha CC, Jorge MT, Ribeiro LA. Snakebites in southern Minas Gerais State, Brazil. $J$ Venom Anim Toxins 7: 56-68, 2001.

9. Jorge MT, Ribeiro LA. Epidemiologia e quadro clínico do acidente por cascavel sul-americana (Crotalus durissus). Rev Inst Med Trop São Paulo 34: 347-354, 1992.

10. Lima JS, Martelli-Júnior H, Martelli DRB, Silva MS, Carvalho SFG, Canela JR, Bonan PRF. Perfil dos acidentes ofídicos no norte do estado de Minas Gerais, Brasil. Rev Soc Bras Med Trop 42: 561-564, 2009.

11. Oliveira FN, Brito MT, Morais ICO, Fook SML, Albuquerque HN. Accidents caused by Bothrops and Bothropoides in the state of Paraíba: epidemiological and clinical aspects. Rev Soc Bras Med Trop 43: 662-667, 2010.

12. Pinho FMO, Oliveira ES, Faleiros F. Acidente ofídico no estado de Goiás. Rev Assoc Med Bras 50: 93-96, 2004.

13. Pinho FO, Vidal EC, Burdmann EA. Atualização em insuficiência renal aguda: insuficiência renal aguda após acidente crotálico. J Bras Nefrol 22: 162-168, 2000.

14. Ribeiro LA, Jorge MT. Acidente por serpente do gênero Bothrops: série de 3.139 casos. Rev Soc Bras Med Trop 30: 475-480, 1997.

15. Sociedade Brasileira de Herpetologia. Brazilian reptiles - list of species 2011. Disponivel em http:// www.sbherpetologia.org.br/?page_id=629. Acessado em janeiro de 2012.

16. Tribunal de Contas do Estado do Rio de Janeiro, Secretaria-Geral de Planejamento. Estudo socioeconômico 2007 Valença. Caracterização do município, 2007. p.7-36.

17. Wen FH, Cardoso JLC, Málaque CMS, França FOS, Sant'anna SS, Fernandes W, Furtado MF, Franco FL, Albuquerque MJ, Kronca FJ, Nalon MA, Rúbio GBG, Silva EM, Leite JCM. Influência das alterações ambientais na epidemiologia dos acidentes ofídicos e distribuição geográfica das serpentes de importância médica nos estados de São Paulo e Paraná, 1988-1997. Informe Epidemiológico do SUS 11: 45-47, 2002. 